\title{
Use of PET/MRI for identification and characterisation of liver lesions
}

\author{
Afshin Nasoodi $\cdot$ Rizwan Syed $\cdot$ Asim Afaq $\cdot$ \\ Jamshed Bomanji
}

Received: 7 November 2013/ Accepted: 13 February 2014/Published online: 22 March 2014

(C) Italian Association of Nuclear Medicine and Molecular Imaging 2014

\begin{abstract}
Magnetic resonance imaging (MRI) has long been considered the gold standard for evaluation of hepatic lesions. There has, however, been much recent interest in functional analysis of malignant lesions, and conventional imaging tests are no longer able to answer all the clinical questions. In the current era, anatomical characterisation, e.g. regarding size and density, is no longer deemed adequate. Combined imaging with PET/CT provides clarity and insight but at times can be challenging, especially in certain parts of the body such as the liver, pelvis and brain, where test sensitivity is compromised. PET/MRI, a hybrid imaging technology, can be used to improve upon the soft-tissue contrast offered by MRI and the biochemical and metabolic information provided by PET, with overall improvement in test sensitivity and hence increased diagnostic accuracy and improved tissue characterisation. Furthermore, various radiolabelled PET tracers can be used to improve the accuracy of staging, determination of the extent of disease, evaluation of treatment response, prognostic assessment and differentiation between post-therapeutic changes and tumour recurrence. In this article, we review the advantages of PET/MRI over conventional imaging in identifying and characterising liver lesions.
\end{abstract}

Keywords PET $\cdot$ MR $\cdot$ Liver $\cdot$ NET $\cdot$ Metastasis

Color figures online at http://link.springer.com/article/10.1007/ s40336-014-0057-z

A. Nasoodi

Nuclear Medicine Department, Imaging Center, Royal Victoria Hospital, Belfast BT12 6BA, UK

R. Syed $(\bowtie) \cdot$ A. Afaq $\cdot$ J. Bomanji

Institute of Nuclear Medicine, University College London

Hospital, London NW1 2BU, UK

e-mail: rizwan.syed@uclh.nhs.uk

\section{Introduction}

The aim of diagnostic imaging, particularly in the setting of a previous history of tumour, is lesion characterisation, allowing differentiation of benign from malignant lesions. This important step in the diagnostic process underpins the subsequent management of the patient. Magnetic resonance imaging (MRI) has long been considered the gold standard for the evaluation of hepatic lesions. There has, however, been much recent interest in functional analysis of malignant lesions since the conventional imaging tests are no longer able to answer all the clinical questions.

The liver is a common site of metastatic disease owing to its rich blood supply; there are occasional cases in which a benign lesion in the liver presents with atypical features, necessitating further evaluation [1]. Targeted treatment is now available for isolated hepatic disease [2] but successful treatment depends on accurate assessment of the lesion, the extent of the liver disease, and the absence of extrahepatic metastases [3].

The concept of combining functional with morphological data during a single acquisition has been the subject of much research conducted with the objective of improving detectability and increasing diagnostic performance. Metabolic imaging with positron emission tomography (PET) combined with computed tomography (CT) [4], and more recently MRI [5], has enabled the acquisition of detailed fused functional and morphological data.

MRI offers high-resolution structural data, superb softtissue contrast and a range of acquisition techniques capable of generating functional data. PET/MRI, a hybrid imaging technology, can be used to improve upon the softtissue contrast offered by MRI and the biochemical and metabolic information provided by PET, with overall improvement in test sensitivity and hence increased 
diagnostic accuracy and improved tissue characterisation. Although much of the experience has been with [ ${ }^{18-}$ F]fluorodeoxyglucose $\left({ }^{18} \mathrm{~F}\right.$-FDG) [1, 6], various radiolabelled PET tracers may be used to improve accuracy of staging, determination of extent of disease, evaluation of treatment response, prognostic assessment and differentiation between post-therapeutic changes and tumour recurrence [7]. The value of PET/MRI will, however, need to be proven through validation of its applications and its overall impact on patient care [7].

The new technology of integrated PET/MRI within a single hardware device [8] allows investigation of a range of tumour biomarkers, such as those for proliferation, apoptosis, angiogenesis, metabolism and hypoxia, using various acquisition techniques and radiotracers. This can result in sophisticated in vivo detection techniques that permit better lesion characterisation at cellular and subcellular levels [9].

This paper reviews the advantages and limitations of using PET/MRI, in comparison with conventional imaging, for the identification and characterisation of liver lesions by highlighting the strengths of its individual components, MRI and PET. Colorectal cancer (CRC) is chosen as a model for imaging of liver metastasis owing to the extensive available literature and the fact that liver disease is commonly encountered in association with this common malignancy. Furthermore, the application of PET/MRI for imaging of metastatic neuroendocrine lesions in the liver is addressed. Reference is also made to what the future could hold in terms of further development of hepatic imaging.

\section{MRI as a powerful partner to PET in imaging of the liver}

Liver MRI is considered to be more accurate for detection and characterisation of focal lesions than $\mathrm{CT}$ or ultrasound (US) [10]. It is commonly used to evaluate indeterminate focal hepatic lesions detected on other imaging studies and to image patients with contraindications to iodinated contrast material.

MRI, using a multitude of imaging parameters, allows a thorough assessment of the hepatobiliary system. Magnetic resonance cholangiopancreatography is a very useful tool in the non-invasive investigation of pathologies related to the biliary tree and pancreas. The sequences rely heavily on T2-weighted images to demonstrate these fluid-filled structures [11].

Liver MRI protocols commonly use a combination of T1- and T2-weighted imaging. Inversion recovery images increase the sensitivity for detecting lesions [10]. Furthermore, out-of-phase imaging can be used to further characterise fat-containing benign lesions, which lose signal on out-of-phase images as compared to in-phase images [12].

T1-weighted post-contrast imaging is very useful for the characterisation of focal hepatic lesions. The choices of contrast include extracellular gadolinium-containing contrast agents, reticuloendothelial contrast agents and hepatocellular-specific contrast agents [13]. Dynamic contrastenhanced (DCE)-MRI using gadolinium-based contrast agents is helpful in differentiating benign from malignant liver lesions and in assessing the vascularity of metastases. Perfusion MRI has even shown high sensitivity for the detection of micro-metastases in the liver, which are associated with a poorer prognosis [14]. While pre-existing liver disease may influence the analysis of dynamic imaging, this has been observed only in animal models [15]. Superparamagnetic iron oxide (SPIO), which belongs to another category of contrast agents, is taken up by the hepatic reticuloendothelial system (RES), resulting in a generalised loss of signal. As a result, hyperintense hepatic malignancies on T2-weighted images become more apparent following contrast administration. Good results have also been achieved in characterising lesions containing functional RES cells, such as focal nodular hyperplasia (FNH) [12]. But it is the third group, liver-specific contrast agents, that has caused most excitement in studies investigating the use of contrast-enhanced MRI for assessment of focal liver lesions. Enhanced MRI using the hepatocellular-specific agent, gadolinium-ethoxybenzyl-diethylenetriamine penta-acetic acid (Gd-EOB-DTPA), is a highly accurate method for the detection and characterisation of liver lesions [13].

The sequence used in liver MRI is diffusion-weighted imaging (DWI), which does not involve contrast administration, but instead relies on Brownian motion of water molecules in the tissue [16]. It is relatively quick to perform and widely used in the clinical setting for disease evaluation in the liver. Restricted water diffusion in cellular tissues gives rise to high signal intensity on DWI-MRI and, upon quantitative evaluation, a lower apparent diffusion coefficient. The evidence suggests that the use of DWI-MRI can substantially improve the sensitivity for detection and identification of liver metastases [14].

DWI-MRI and DCE-MRI with extracellular gadolinium have been used for the diagnosis of liver metastases [16] and the two may be employed interchangeably. Since restricted diffusion is not exclusive to malignant lesions [14], the information obtained from DWI-MRI cannot be used in isolation to differentiate a malignant from a benign lesion. Use of DWI-MRI in conjunction with hepatocellular-specific contrast-enhanced MRI may be a solution. Although Gd-EOB-DTPA-enhanced MRI has shown better performance than DWI in the detection and 
characterisation of focal liver lesions, using the two techniques together offers increased confidence in the final diagnosis, hence justifying additional DWI sequences in a dedicated MRI protocol using hepatocyte-specific contrast media [16]. Furthermore, Gd-EOB-DTPA-enhanced MRI can be used in the characterisation of benign solid liver lesions such as FNH and adenomas [13]. Overall, Gd-EOBDTPA-enhanced MRI and DWI-MRI are complementary. Small lesions adjacent to the liver vessels may be better seen on DWI-MRI. Conversely, small metastases adjacent to the diaphragm or in the left hepatic lobe may be obscured by motion artefacts or magnetic field inhomogeneity on DWI-MRI, but better illustrated with Gd-EOBDTPA-enhanced MRI [16].

\section{${ }^{18}$ F-FDG PET/CT with and without contrast in liver lesions}

In recent years, DCE-CT has been an essential imaging tool for the detection of liver metastases [17]. It has been shown that by demonstrating arterialisation of flow within the liver, it is possible to confirm the presence of a tumour in the area of interest [14].

Functional imaging with ${ }^{18} \mathrm{~F}-\mathrm{FDG}$ PET has been reported to show higher sensitivity and specificity compared with CT for the detection of viable liver metastases following targeted treatment [18]. Since its introduction approximately a decade ago, PET/CT has become an important imaging tool in patients with oncological conditions [17]. The reported sensitivity of US, CT, MRI and FDG PET in hepatic metastases originating from colorectal, gastric and oesophageal cancers has been reported to be $55,72,76$ and $90 \%$, respectively [19]. Optimal detection and characterisation of liver lesions require the use of fused contrast-enhanced PET/CT owing to suboptimal sensitivity of low-dose unenhanced PET/CT [3]. The unenhanced technique is, however, preferred in patients with contrast allergy or poor renal function.

The liver is a common site of metastasis in CRC, in which there is an approximately $50 \%$ chance of liver disease within 5 years despite curative primary resection. ${ }^{18}$ F-FDG PET/CT is routinely used in the assessment of the liver in patients with CRC hepatic metastasis. In the absence of disease elsewhere, hepatic resection offers suitable patients improved survival [20]. There is, however, a potential pitfall with PET/CT, in that, compared with MRI, it has a relatively low sensitivity for the detection of liver metastases smaller than $1 \mathrm{~cm} \mathrm{[21].}$ Research has illustrated that MRI and contrast-enhanced PET/CT are the best tests for detection of liver lesions, with MRI performing better in the characterisation of metastases [3].

\section{Where does PET/MRI stand in the detection and characterisation of liver lesions?}

Hybrid PET/MRI systems are now commercially available as a result of the development of the whole-body MRI technique, technological advances in the manufacturing of MRI-compatible PET detectors, and the ability to carry out attenuation correction of the PET data using the Dixon sequence on MRI [22]. This novel imaging modality is thought to play an important role in liver imaging, in which both high soft-tissue resolution and functional information are required. One of the greatest advantages of PET/MRI over PET/CT is the simultaneous data acquisition, enabling the achievement of excellent soft-tissue contrast, very good spatial resolution and very accurate temporal and spatial image fusion. Additionally, functional MRI, such as arterial spin labelling or spectroscopy, can be performed with PET/MRI [23]. Occasionally, assessment of a liver lesion suspicious for a metastasis may require both ${ }^{18} \mathrm{~F}$-FDG PET/ $\mathrm{CT}$ and MRI in the pre-surgical planning phase [24]. PET/ MRI can offer a clinically competitive solution in terms of cost-effectiveness and patient acceptance, with a reasonable examination time, allowing increased workflow and patient throughput [25].

PET/MRI provides an impressive fusion of high-resolution anatomy and metabolic activity in a one-stop shop examination, which is particularly valuable in paediatrics, and obviates the need for multiple hybrid imaging, otherwise often required in patients with oncological disease. This helps to reduce the ionising radiation dose to patients. The radiation exposure in PET/MRI is from the PET component and it is $3-4 \mathrm{mSv}[26,27]$. The typical effective dose for a child undergoing diagnostic $\mathrm{PET} / \mathrm{CT}$ is about $25 \mathrm{mSv}$ [28]. Thus there is $80 \%$ less radiation exposure from a hybrid PET/MRI compared with a PET/CT study [29]. Finally, in children, the intensity of ${ }^{18} \mathrm{~F}$-FDG uptake in neuroblastoma is thought to be an adverse prognostic factor. ${ }^{18} \mathrm{~F}$-FDG PET is recommended in addition to MRI and metaiodobenzylguanidine scintigraphy for the initial evaluation of highrisk neuroblastoma with small liver and lung metastases. It may be concluded that the combination of PET and MRI offers complementary information regarding the presence of small lesions and the degree of tumour differentiation. When the two methods are concordant, the confidence in identification of small lesions is improved, and PET/MRI offers clear advantages over either modality alone [29].

PET/MRI has been found to outperform PET/CT in terms of conspicuity of liver lesions, with better sensitivity and specificity [12]. Overall, co-registered PET and unenhanced MR images have been shown to outperform unenhanced PET/CT in the imaging of PET-positive liver 

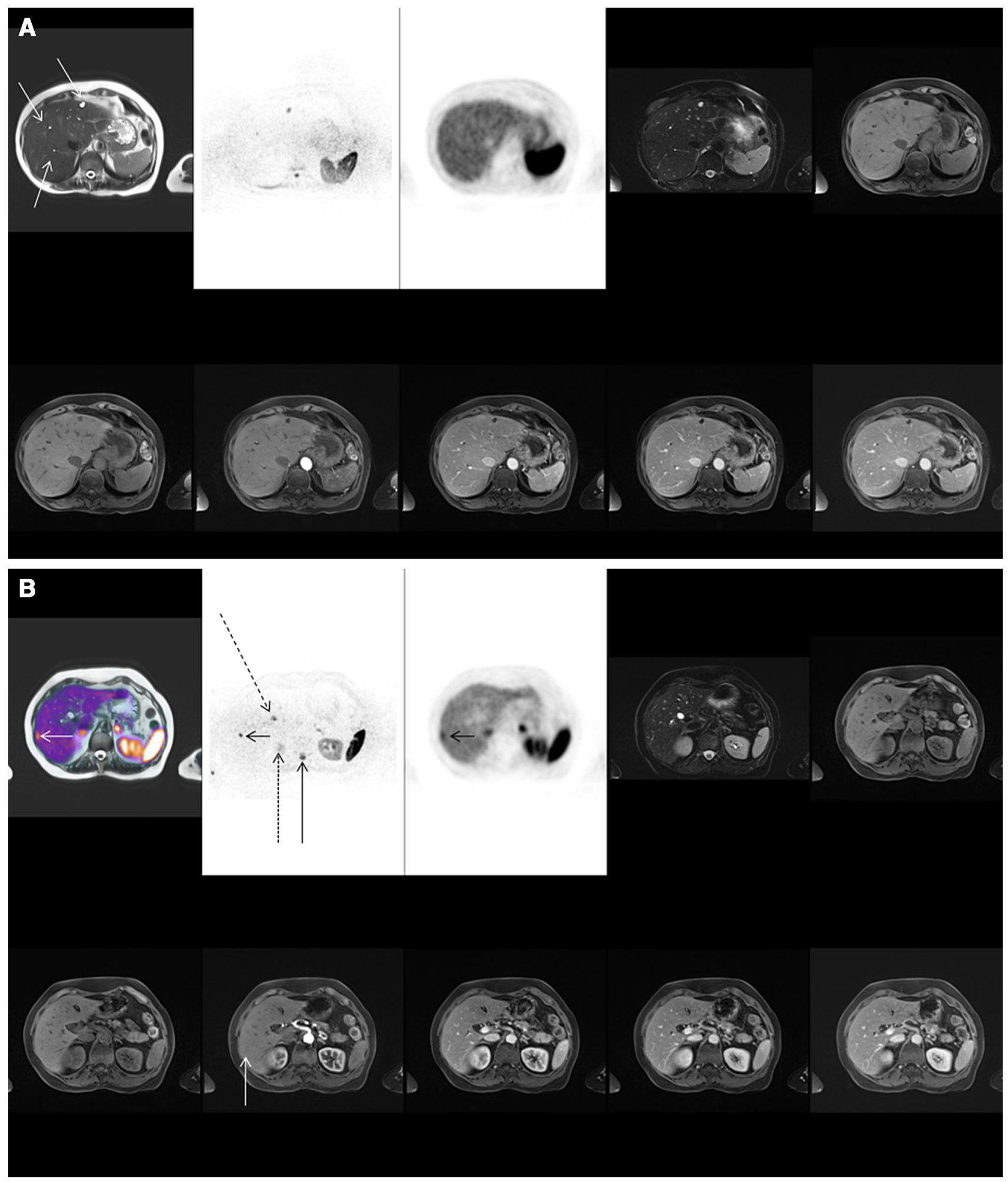

lesions, with better delineation of small lesions as well as reliable localisation of lesions to the corresponding liver segment $[5,12]$.
Just as importantly, the standardised uptake value (SUV) derived from PET/MRI can be used reliably in the clinical setting [30, 31]. 
४Fig. 1 Ga DOTATATE PET/MRI in metastatic NETs. This study shows a combination of simple hepatic cysts and a peripheral focal lesion in the right hepatic lobe which is tracer avid and demonstrates rim enhancement in keeping with a metastatic deposit. a Simple cysts but no metastases. 1st row: T2 HASTE (white arrows show cysts), inverted b800 diffusion-weighted image, Ga DOTATATE PET, fatsuppressed T2 axial, pre-contrast T1 axial. 2nd row: sequential T1 contrast series. The cysts are hyperintense on $\mathrm{T} 2$, and are seen on the DWI, despite not being of increased cellularity due to the 'T2 shine through effect'. There is no increased uptake on the PET images and no enhancement on the contrast MRI series. b Right hepatic lobe metastasis in the presence of simple cysts. 1st row: fused T2 HASTE PET/MRI, inverted b800 diffusion-weighted image, Ga DOTATATE PET, fat-suppressed $\mathrm{T} 2$ axial, pre-contrast $\mathrm{T} 1$ axial 2nd row: sequential T1 contrast series. The white arrow on the top left image shows the metastasis. On the diffusion-weighted image, the short black arrow shows that the metastasis is hypercellular. Other areas which are bright on DWI (dark on this inverted image) and do not represent disease include partially images upper pole of the right kidney (square dotted arrow), a cyst in the right hepatic lobe (dashed arrow) and CSF. The white arrow on the second image on the second row indicates mild rim enhancement of the metastasis (Color figure online)

Our PET/MRI protocol is performed as a complementary combination of whole-body imaging to determine the spread of the cancer and includes additional regional MRI to provide better characterisation of primary disease.

The contrast-enhanced MRI technique using all three categories of contrast media can be adopted in combined PET/MRI [12]. Dynamic gadolinium-enhanced T1-GRE imaging depicts the pattern of enhancement of hepatic neoplasms, which is very useful for the accurate characterisation of focal hepatic lesions, with incremental value being achieved through addition of quantitative data from both PET and MRI [12].

The commonly encountered artefacts on stand-alone MRI and PET studies can be tackled satisfactorily when imaging with integrated PET/MRI systems. First, both MRI and PET are lengthy studies and hence susceptible to respiratory motion artefacts with the possibility of misregistration of the lesions, which can lead to a "smearing" effect of the tracer uptake [32]. The use of respiratory triggering or phase reordering can minimise any significant respiratory-related motion artefacts that may be present. Second, the implementation of MR-based motion-correction technologies to correct for gross patient motion and breathing in view of the relatively long PET data acquisition times seems promising [33]. Third, the susceptibility artefacts in DWI can be minimised by using half-Fourier acquisition single-shot turbo spin echo (HASTE) techniques [34]. Finally, T1-weighted GRE images may contain pulsation artefacts from the aorta that can be misinterpreted as a focal lesion in the left lobe of the liver [10]. Such artefacts can be easily recognised on a PET/MRI study by virtue of the absence of metabolic activity as revealed by lack of ${ }^{18}$ F-FDG uptake by the artefact.
By combining the functional information from ${ }^{18} \mathrm{~F}$ FDG PET with the anatomical information from MRI, ${ }^{18}$ F-FDG PET/MRI is capable of offering high sensitivity and specificity in the detection of liver metastases and better detection of extrahepatic disease in the same examination [12]. Furthermore, the MRI component of PET/MRI is considered the imaging modality of choice for the assessment of lesions in the presence of preexisting chronic liver disease. PET/MRI is likely to increase the diagnostic confidence when compared with Gd-EOB-DTPA-enhanced MRI. Regardless of the size of the focal liver lesion, PET/MRI is generally more sensitive and specific than PET/CT (reported sensitivity and specificity of up to 93 and $96 \%$, respectively, for PET/ MRI vs 76 and $85 \%$, respectively, for PET/CT [32]) and offers significantly higher diagnostic confidence [21]. PET/MRI can be expected to display higher accuracy than PET/CT when assessing the liver for metastatic spread. An additional potential advantage of PET/MRI, due to the inherently high-resolution anatomical images it provides, may be increased specificity in characterising small, indeterminate lesions found on MRI [32].

PET/MRI is likely to perform well in the assessment of benign focal liver lesions. Benign liver lesions have a high prevalence and can be differentiated with variable success using anatomical techniques [1, 35]. Functional imaging using PET and DWI-MRI is increasingly needed for liver lesion assessment. Furthermore, DWI may be useful in the assessment of suspicious, normal-sized, ${ }^{18}$ F-FDG-negative portal nodes [34]. Diffusion-weighted sequences can be performed with PET/MRI and heavily T2-weighted sequences can help to identify cystic lesions and haemangioma [36]. Benign focal lesions such as simple cysts, abscesses, FNH, adenomas and haemangiomas are easily recognised with the MRI component of the study [37]. In addition, atypical haemangiomas, FNH and adenomas are all usually associated with low or no FDG uptake on combined PET/MRI [38]. Furthermore, a potential role of PET in differentiating adenomas from $\mathrm{FNH}$ has been suggested in a small prospective study, with SUVs of 1.22 or higher for FNH when using the tracer ${ }^{18} \mathrm{~F}$-fluorocholine [39].

Primary liver malignancies are less commonly encountered compared with metastatic and benign lesions. The available data are supportive of an important role for PET/ MRI in patients with primary liver malignancies. Hepatocellular carcinoma (HCC) may be evaluated by DCE-MRI. While there is evidence that detection and characterisation of HCC can be significantly improved when imaging is performed using ${ }^{18} \mathrm{~F}$-choline PET/CT [40], in our experience the detection of HCC has been poor. Despite this, one may infer that radiopharmaceuticals other than ${ }^{18} \mathrm{~F}-\mathrm{FDG}$ may represent an attractive option and, overall, might make 


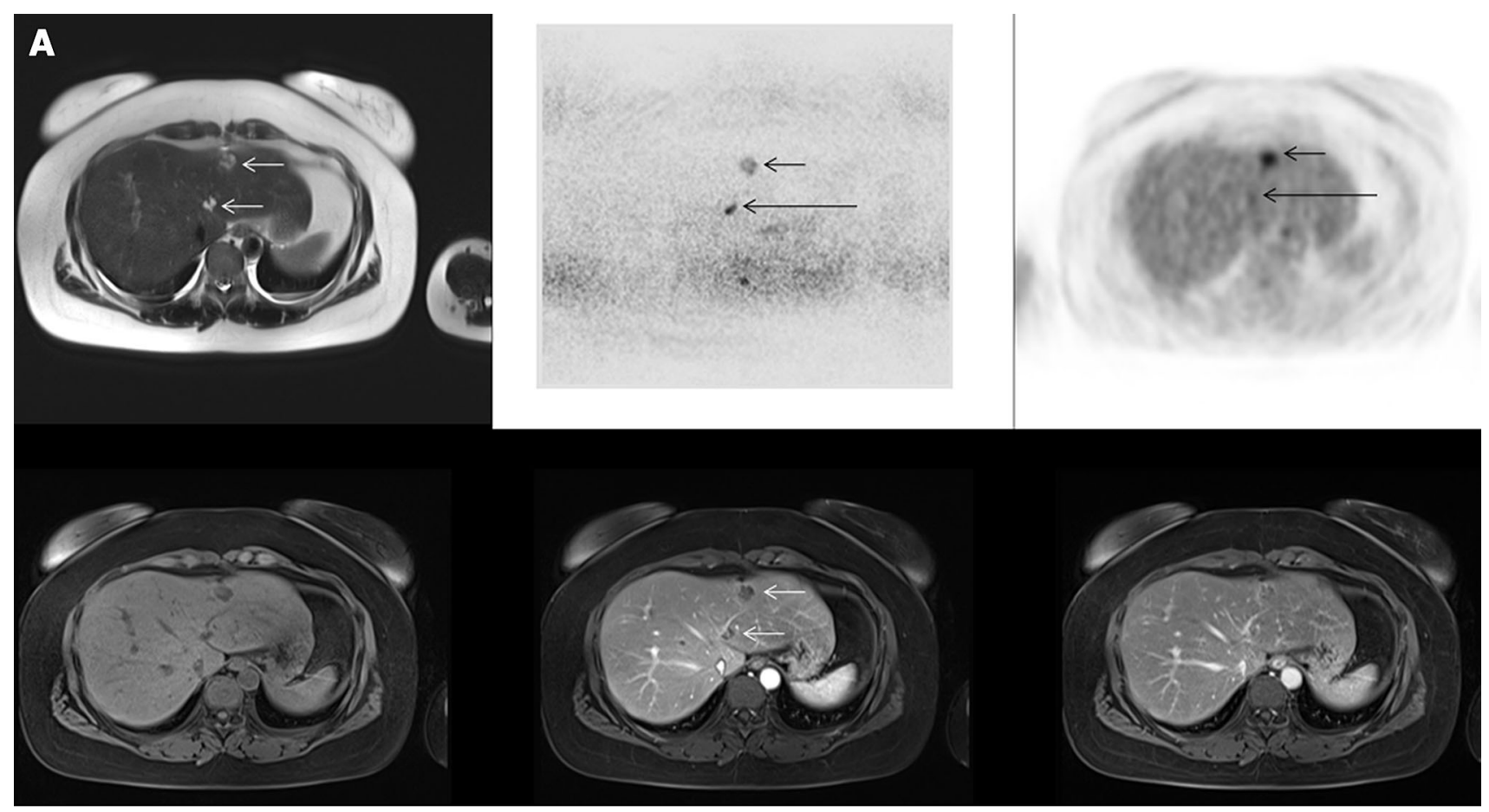

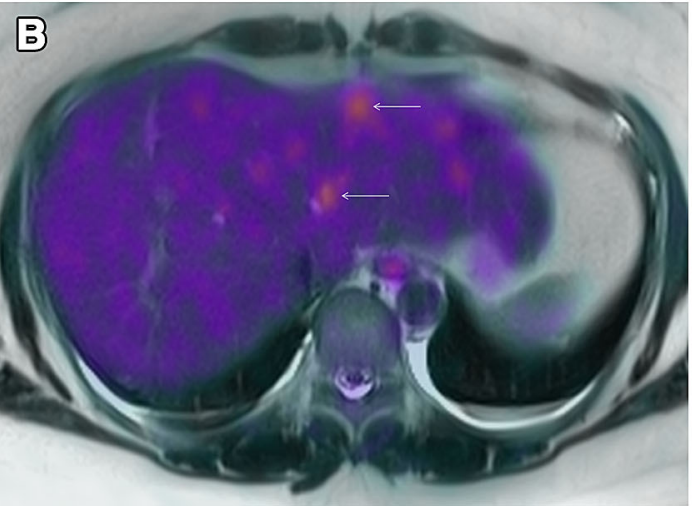

Fig. 2 FDG PET/MRI in metastatic colorectal cancer. a 1st row, T2 HASTE, inverted b800 DWI, FDG PET. 2nd row, pre- and post-contrast T1 axial images. White arrows show metastases. b Fused T2 HASTE and FDG PET. White arrows show metastases. The small metastases are seen in segment 4 and adjacent to the left hepatic vein, with peripheral enhancement and increased FDG uptake. The lesions are partly treated and cystic (bright on T2). This causes increased signal on DWI (T2

PET/MRI a more accurate test in the diagnosis and characterisation of liver lesions.

The wider use of PET/MRI scanners will be greatly fostered by the use of agents that incorporate both PET isotopes and MRI contrast agents. Research work has been successful in developing combined probes, such as SPIOcoated PET tracers, that show great potential for use in cell tracking with PET/MRI [25, 41, 42].

Dedicated 7T liver MRI has revealed potential benefits with promising unenhanced angiographic applications and it may be used in combined PET/MRI in the future [36]. Finally, there has been a re-emergence of interest in shine through). However, there is also some residual tumour, as can be seen on the PET images. The more anterior of the two lesions is less bright on DWI (dark on this inverted image) but demonstrates more uptake of FDG relative to the more posterior lesion. This is because the posterior lesion is more cystic (post-treatment) and contains less active tumour, but appears more intense on the DWI image due to the T2 shine through effect (Color figure online)

dynamic PET, but the significance of this technique in liver imaging is unknown and multicentre studies would be key in determining its clinical value for quantitative imaging [43].

\section{PET/MRI for imaging of NET hepatic metastases}

Neuroendocrine tumours (NETs) are a rare group of neoplasms most commonly originating from the pancreas and terminal ileum [44]. They have characteristically been associated with overexpression of somatostatin receptor 
subtype 2, which has long been exploited for imaging of these tumours using the somatostatin analogue, octreotide [45]. The liver is the most common site for metastasis [46], and liver metastases are associated with worse 5-year survival [47]. PET with somatostatin receptor analogues such as ${ }^{68}$ Ga-labelled 1,4,7,10-tetra-azacyclododecane1,4,7,10-tetra-acetic acid (DOTA) peptides, which include DOTA-D-Phe ${ }^{1}$-Tyr $^{3}$-octreotide (DOTATOC), DOTA-1$\mathrm{Nal}^{3}$-octreotide (DOTANOC), or DOTA-D-Phe ${ }^{1}-\mathrm{Tyr}^{3}$ octreotate (DOTATATE), has shown higher diagnostic potential because of a higher affinity for the somatostatin receptor subtype 2 [48]. ${ }^{68}$ Ga-DOTATOC PET/MRI imaging of focal liver lesions has yielded promising results based on better contrast resolution and detectability. Additional diagnostic MR protocols may offer further benefits. Fused ${ }^{68}$ Ga-DOTATOC PET/Gd-EOB-DTPAenhanced MRI is more sensitive than PET and more specific than MRI [49] for identifying and characterising liver lesions and predicting a favourable response to peptide receptor radionuclide therapy. PET/MRI has a higher sensitivity and specificity (91 and $96 \%$, respectively) than PET/CT (74 and $88 \%$, respectively) for the detection of liver metastases [32]. The differential uptake by liver lesions of ${ }^{68} \mathrm{Ga}$-DOTA-peptide PET/CT and ${ }^{18} \mathrm{~F}$-FDG PET/ $\mathrm{CT}$ is valuable in lesion characterisation [50]. ${ }^{18} \mathrm{~F}-\mathrm{FDG}-$ positive, ${ }^{68} \mathrm{Ga}$-DOTA-peptide-negative status of the lesion heralds poor differentiation and aggressive behaviour. ${ }^{68} \mathrm{Ga}$-PET/CT is currently considered the standard imaging modality in the management of NETs [51] (Figs. 1, 2).

Available data suggest that ${ }^{68} \mathrm{Ga}$-DOTATOC PET/MRI and ${ }^{68} \mathrm{Ga}$-DOTATOC PET/CT are of similar diagnostic value in patients with gastroenteropancreatic NETs [52]. PET/MRI is thought to perform better in the characterisation of small indeterminate lesions, in the presence of contraindications to the use of contrast media [53].

\section{Conclusions}

PET/MRI is a versatile imaging tool which can improve anatomical localisation and characterisation of hepatic lesions.

PET/CT will remain the workhorse of cancer imaging until PET/MRI is in wide clinical use and until more clinical data become available. In liver imaging, PET/MRI is superior to stand-alone PET/CT or MRI. PET/MRI can be performed without contrast media and is associated with reduced ionising radiation. The availability of protean functional imaging techniques and multiple contrast media makes PET/MRI a formidable imaging tool. The diversity of PET tracers adds further specificity to PET/MRI. In order for it to be viable in clinical routine, however, the imaging workflow and use of various sequences must be based on the specific clinical needs of patients in order to maximise useful information whilst minimising acquisition time. A definite killer application has not yet been defined for PET/MRI, but in the authors' opinion this is just a matter of time as the potential is obvious. The next decade will witness the development of more technologically advanced combined PET/MRI machines with further innovations to suit the era of personalised patient management. In the meantime, however, PET/MRI remains a prominent research tool for answering complex clinical questions in a selective manner.

Acknowledgments This work was in part supported by the National Institute for Health Research University College London Hospitals Biomedical Research Centre.

Ethical standard All procedures followed were in accordance with the ethical standards of the responsible committee on human experimentation and with the Helsinki Declaration of 1975, as revised in 2008. Informed consent was obtained from all patients included in the study.

Conflict of interest None of the authors (Dr A. Nasoodi, Dr R. Syed, Dr A. Afaq, Dr J. Bomanji) have any conflict of interest.

\section{References}

1. Sharma B, Martin A, Zerizer I (2013) Positron emission tomography-computed tomography in liver imaging. Semin Ultrasound CT MR 34:66-80

2. Desar IM, van Herpen CM, van Laarhoven HW, Barentsz JO, Oyen WJ, van der Graaf WT (2009) Beyond RECIST: molecular and functional imaging techniques for evaluation of response to targeted therapy. Cancer Treat Rev 35:309-321

3. Cantwell CP, Setty BN, Holalkere N, Sahani DV, Fischman AJ, Blake MA (2008) Liver lesion detection and characterization in patients with colorectal cancer: a comparison of low radiation dose non-enhanced PET/CT, contrast-enhanced PET/CT, and liver MRI. J Comput Assist Tomogr 32:738-744

4. Townsend DW, Beyer TA (2002) Combined PET/CT scanner: the path to true image fusion. Br J Radiol 75:s24-s30

5. Drzezga A, Souvatzoglou M, Eiber M et al (2012) First clinical experience with integrated whole-body PET/MR: comparison to $\mathrm{PET} / \mathrm{CT}$ in patients with oncologic diagnoses. J Nucl Med 53:845-855

6. Abouzied MM, Crawford ES, Nabi HA (2005) ${ }^{18}$ F-FDG imaging: pitfalls and artifacts. J Nucl Med Technol 33:145-155

7. Judenhofer MS, Cherry SR (2013) Applications for preclinical PET/MRI. Semin Nucl Med 43:19-29

8. Delso G, Furst S, Jakoby B, Ladebeck R, Ganter C, Nekolla SG, Schwaiger M, Ziegler SI (2011) Performance measurements of the Siemens mMR integrated whole-body PET/MR scanner. J Nucl Med 52:1914-1922

9. Sauter AW, Wehrl HF, Kolb A, Judenhofer MS, Pichler BJ (2010) Combined PET/MRI: one step further in multimodality imaging. Trends Mol Med 16:508-515

10. Coenegrachts K (2009) Magnetic resonance imaging of the liver: new imaging strategies for evaluating focal liver lesions. World $\mathbf{J}$ Radiol 31:72-85 
11. Griffin N, Yu D, Grant LA (2013) Magnetic resonance cholangiopancreatography: pearls, pitfalls, and pathology. Semin Ultrasound CT MRI 34:32-43

12. Kuhn FP, Crook DW, Mader CE, Appenzeller P, von Schulthess GK, Schmid DT (2013) Discrimination and anatomical mapping of PET-positive lesions: comparison of CT attenuation-corrected PET images with coregistered MR and CT images in the abdomen. Eur J Nucl Med Mol Imaging 40:44-51

13. Ringe K, Husarik DB, Sirlin CB, Merkle EM (2010) Gadoxetate disodium-enhanced MRI of the liver: part 1, protocol optimization and lesion appearance in the non cirrhotic liver. AJR Am J Roentgenol 195:13-28

14. Goh V, Gourtsoyianni S, Koh D-M (2013) Functional imaging of the liver. Semin Ultrasound CT MRI 34:54-65

15. Saito S, Obata A, Kashiwagi Y, Abe K, Murase K (2013) Dynamic contrast-enhanced MRI of the liver in Mrp2-deficient rats using the hepatobiliary contrast agent Gd-EOB-DTPA. Invest Radiol 48:548-553

16. Donati OF, Fischer MA, Chuck N, Hunziker R, Weishaupt D, Reiner CS (2013) Accuracy and confidence of Gd-EOB-DTPA enhanced MRI and diffusion-weighted imaging alone and in combination for the diagnosis of liver metastases. Eur J Radiol $82: 822-828$

17. Buchbender C, Heusner TA, Lauenstein TC, Bockisch A, Antoch G (2012) Oncologic PET/MRI, Part 1: tumors of the brain, head and neck, chest, abdomen, and pelvis. J Nucl Med 53:1244-1252

18. Veit P, Antoch G, Stergar H, Bockisch A, Forsting M, Kuehl H (2006) Detection of residual tumor after radiofrequency ablation of liver metastasis with dual-modality PET/CT: initial results. Eur Radiol 16:80-87

19. Kinkel K, Lu Y, Both M, Warren RS, Thoeni RF (2002) Detection of hepatic metastases from cancers of the gastrointestinal tract by using noninvasive imaging methods (US, CT, MR imaging, PET): a meta-analysis. Radiology 224:748-756

20. Pathak S, Jones R, Tang JM et al (2011) Ablative therapies for colorectal liver metastases: a systematic review. Colorectal Dis 13:e252-e265

21. Donati OF, Hany TF, Reiner CS et al (2010) Value of retrospective fusion of PET and MR images in detection of hepatic metastases: comparison with 18F-FDG PET/CT and Gd-EOBDTPA-enhanced MRI. J Nucl Med 51:692-699

22. Beyer T, Moser E (2013) MR/PET or PET/MRI: does it matter? MAGMA 26:1-4

23. Pichler BJ, Judenhofer MS, Pfannenberg C (2008) Multimodal imaging approaches: PET/CT and PET/MRI. Handb Exp Pharmacol 185:109-132

24. Kalemis A, Delattre BMA, Heinzer S (2013) Sequential wholebody PET/MR scanner: concept, clinical use, and optimisation after 2 years in the clinic. The manufacturer's perspective. MAGMA 26:5-23

25. Yankeelov TE, Peterson TE, Abramson RG et al (2012) Simultaneous PET-MRI in oncology: a solution looking for a problem? Magn Reson Imaging 30:1342-1356

26. Stauss J, Franzius C, Pfluger $\mathrm{T}$ et al, European Association of Nuclear Medicine (2008). Guidelines for ${ }^{18}$ F-FDG PET and PETCT imaging in paediatric oncology. Eur J Nucl Med Mol Imaging 35:1581-1588

27. Fahey FH, Treves ST, Adelstein SJ (2011) Minimizing and communicating radiation risk in pediatric nuclear medicine. J Nucl Med 52:1240-1251

28. Chawla SC, Federman N, Zhang D et al (2010) Estimated cumulative radiation dose from $\mathrm{PET} / \mathrm{CT}$ in children with malignancies: a 5-year retrospective review. Pediatr Radiol 40:681-686

29. Hirsch FW, Sattler B, Sorge I et al (2013) PET/MR in children. Initial clinical experience in paediatric oncology using an integrated PET/MR scanner. Pediatr Radiol 43:860-875
30. Heusch P, Buchbender C, Beiderwellen K et al (2013) Standardized uptake values for $\left[{ }^{18} \mathrm{~F}\right]$ FDG in normal organ tissues: comparison of whole-body PET/CT and PET/MRI. Eur J Radiol 82:870-876

31. Partovi S, Thomas S, Traughber BJ, Ellis R, Faulhaber P. Preliminary evaluation of dedicated PET/MRI in gastrointestinal malignancy: Qualitative and quantitative comparison to PET/CT. Pract Radiat Oncol April-June Supplement 2013. Poster presentation

32. Antoch G, Bockisch A (2009) Combined PET/MRI: a new dimension in whole-body oncology imaging? Eur J Nucl Med Mol Imaging 36(Suppl 1):S113-S120

33. Quick HH, von Gall $C$, Zeilinger $M$ et al (2013) Integrated whole-body PET/MR hybrid imaging. Invest Radiol 48:280-289

34. Buchbender $\mathrm{C}$, Hartung-Knemeyer $\mathrm{V}$, Beiderwellen $\mathrm{K}$ et al (2013) Diffusion-weighted imaging as part of hybrid PET/MRI protocols for whole-body cancer staging: does it benefit lesion detection? Eur J Radiol 82:877-882

35. Frampas E, Lassau N, Zappa M, Vullierme M-P, Koscielny S, Vilgrain V (2013) Advanced hepatocellular carcinoma: early evaluation of response to targeted therapy and prognostic value of perfusion CT and dynamic contrast enhanced-ultrasound. Preliminary results. Eur J Radiol 82:e205-e211

36. Umutlu L, Bitz AK, Maderwald S et al (2013) Contrast-enhanced ultra-high-field liver MRI: a feasibility trial. Eur J Radiol 82:760-767

37. Burke C, Grant LA, Goh V, Griffin N (2013) The role of hepatocyte-specific contrast agents in hepatobiliary magnetic resonance imaging. Semin Ultrasound CT MRI 34:44-53

38. Vigrain V, Boulos L, Vullierme MP et al (2000) Imaging of atypical hemangiomas of the liver with pathologic correlation. Radiographics 20:379-397

39. Esschert JW, Bieze M, Beuers UH (2011) Differentiation of hepatocellular adenoma and focal nodular hyperplasia using ${ }^{18} \mathrm{~F}$ fluorocholine PET/CT. Eur J Nucl Med Mol Imaging 38:436-440

40. von Schulthess GK, Kuhn FP, Kaufmann P, Veit-Haibach P (2013) Clinical positron emission tomography/magnetic resonance imaging applications. Semin Nucl Med 43:3-10

41. Patel D, Kell A, Simard B, Xiang B, Lin HY, Tian G (2011) The cell labeling efficacy, cytotoxicity and relaxivity of copper-activated MRI/PET imaging contrast agents. Biomaterials 32:1167-1176

42. Yang X, Hong H, Grailer JJ et al (2011) cRGD-functionalized, DOX-conjugated, and ${ }^{64} \mathrm{Cu}$-labeled superparamagnetic iron oxide nanoparticles for targeted anticancer drug delivery and PET/MR imaging. Biomaterials 32:4151-4160

43. Muzi M, O’Sullivan F, Mankoff DA et al (2012) Quantitative assessment of dynamic PET imaging data in cancer imaging. Magn Reson Imaging 30:1203-1215

44. Hauso O, Gustafsson BI, Kidd M et al (2008) Neuroendocrine tumor epidemiology: contrasting Norway and North America. Cancer 113:2655-2664

45. Reubi JC, Maecke HR (2008) Peptide-based probes for cancer imaging. J Nucl Med 49:1735-1738

46. Steinmuller T, Kianmanesh R, Falconi M et al (2008) Consensus guidelines for the management of patients with liver metastases from digestive (neuro)endocrine tumors: foregut, midgut, hindgut, and unknown primary. Neuroendocrinology 87:47-62

47. Rindi G, D'Adda T, Froio E, Fellegara G, Bordi C (2007) Prognostic factors in gastrointestinal endocrine tumors. Endocr Pathol 18:145-149

48. Kumar R, Sharma P, Garg P et al (2011) Role of $\left({ }^{68}\right) \mathrm{Ga}$ DOTATOC PET-CT in the diagnosis and staging of pancreatic neuroendocrine tumours. Eur Radiol 21:2408-2416

49. Schreiter NF, Nogami M, Steffen I, Pape UF, Hamm B, Brenner W, Röttgen R (2012) Evaluation of the potential of PET-MRI 
fusion for detection of liver metastases in patients with neuroendocrine tumours. Eur Radiol 22:458-467

50. Binderup T, Knigge U, Loft A, Mortensen J, Pfeifer A, Federspiel B, Hansen CP, Højgaard L, Kjaer A (2010) Functional imaging of neuroendocrine tumors: a head-to-head comparison of somatostatin receptor scintigraphy, ${ }^{123} \mathrm{I}-\mathrm{MIBG}$ scintigraphy, and ${ }^{18} \mathrm{~F}$ FDG PET. J Nucl Med 51:704-712

51. Schreiter NF, Brenner W, Nogami M et al (2012) Cost comparison of ${ }^{111}$ In-DTPA octreotide scintigraphy and ${ }^{68}$ Ga-DOTATOC PET/CT for staging enteropancreatic neuroendocrine tumours. Eur J Nucl Med Mol Imaging 39:72-82
52. Gaertner FC, Beer AJ, Souvatzoglou M et al (2013) Evaluation of feasibility and image quality of ${ }^{68} \mathrm{Ga}$-DOTATOC positron emission tomography/magnetic resonance in comparison with positron emission tomography/computed tomography in patients with neuroendocrine tumors. Invest Radiol 48:263-272

53. Beiderwellen KJ, Poeppel TD, Hartung-Knemeyer V et al (2013) Simultaneous ${ }^{68} \mathrm{Ga}$-DOTATOC PET/MRI in patients with gastroenteropancreatic neuroendocrine tumors: initial results. Invest Radiol 48:273-279 